\title{
Comparison of Post-injection Site Pain Between Technetium Sulfur Colloid and Technetium Tilmanocept in Breast Cancer Patients Undergoing Sentinel Lymph Node Biopsy
}

\author{
Jonathan T. Unkart, MD ${ }^{1}$, Jennifer L. Baker, MD', Ava Hosseini, MD ${ }^{1}$, Carl K. Hoh, $\mathrm{MD}^{2}$, Mark S. Wallace, $\mathrm{MD}^{3}$, \\ David R. Vera, $\mathbf{P h D}^{2,4}$, and Anne M. Wallace, $\mathrm{MD}^{1}$ \\ ${ }^{1}$ Department of Surgery, University of California, San Diego, La Jolla, CA ; ${ }^{2}$ Department of Radiology, University of \\ California, San Diego, La Jolla, CA; ${ }^{3}$ Department of Anesthesia, University of California, San Diego, La Jolla, CA; \\ ${ }^{4}$ Molecular Imaging Program, University of California, San Diego, La Jolla, CA
}

\begin{abstract}
Background. No prior studies have examined injection pain associated with Technetium-99m Tilmanocept (TcTM).

Methods. This was a randomized, double-blinded study comparing postinjection site pain between filtered Technetium Sulfur Colloid (fTcSC) and TcTM in breast cancer lymphoscintigraphy. Pain was evaluated with a visual analogue scale (VAS) $(0-100 \mathrm{~mm})$ and the short-form McGill Pain Questionnaire (SF-MPQ). The primary endpoint was mean difference in VAS scores at 1-min postinjection between fTcSC and TcTM. Secondary endpoints included a comparison of SF-MPQ scores between the groups at $5 \mathrm{~min}$ postinjection and construction of a linear mixed effects model to evaluate the changes in pain during the 5-min postinjection period.

Results. Fifty-two patients underwent injection (27fTcSC, 25-TcTM). At 1-min postinjection, patients who received fTcSC experienced a mean change in pain of $16.8 \mathrm{~mm}$ (standard deviation (SD) 19.5) compared with $0.2 \mathrm{~mm}$ (SD 7.3) in TcTM $(p=0.0002)$. At $5 \mathrm{~min}$ postinjection, the mean total score on the SF-MPQ was 2.8 (SD 3.0) for fTcSC versus 2.1 (SD 2.5) for TcTM $(p=0.36)$. In the mixed effects model, injection agent $(p<0.001)$, time $(p<0.001)$ and their interaction $(p<0.001)$ were associated with change in pain during the 5 -min postinjection period. The model found fTcSC
\end{abstract}

(C) Society of Surgical Oncology 2015

First Received: 2 June 2015;

Published Online: 15 August 2015

A. M. Wallace, MD

e-mail: amwallace@ucsd.edu resulted in significantly more pain of $15.2 \mathrm{~mm}(p<0.001)$, $11.3 \mathrm{~mm}(p=0.001)$, and $7.5 \mathrm{~mm}(p=0.013)$ at 1,2 , and 3 min postinjection, respectively.

Conclusions. Injection with fTcSC causes significantly more pain during the first 3 min postinjection compared with TcTM in women undergoing lymphoscintigraphy for breast cancer.

Sentinel lymph node (SLN) biopsy is the standard procedure for axillary staging in breast cancer patients with clinically negative lymph nodes. Technetium sulfur colloid (TcSC) is the most commonly used radiotracer for SLN biopsy in breast cancer around the United States. Receiving an injection of TcSC preoperatively is known to cause considerable injection site pain. ${ }^{1}$ Prior studies have explored various methods to decrease pain associated with TcSC injection with mixed results. A study by Stojadinovic et al. demonstrated that adding lidocaine to a mixture with TcSC decreased injection site pain; however, a study by O'Connor et al. found no benefit of applying topical anesthetics before TcSC injection. ${ }^{2,3}$

Technetium-99m tilmanocept (TcTM), a recently FDAapproved radiopharmaceutical designed for SLN identification, travels through lymphatics and binds to the CD206 receptor within macrophages present in lymphatic tissue. ${ }^{4,5}$ During preapproval clinical trials at our institution, nuclear medicine technicians and radiology staff anecdotally observed that patients undergoing TcTM injection reported less pain compared with patients undergoing injection with filtered TcSC (fTcSC). ${ }^{6,7}$ Pain with TcTM injection has not previously been studied in the literature.

The primary goal of this study was to assess the difference in the amount of injection site pain experienced by 
breast cancer patients after receiving an injection of fTcSC versus TcTM prior to SLN imaging and biopsy.

\section{METHODS}

\section{Patients}

This was a randomized, double-blinded, single-institution, controlled clinical trial comparing postinjection site pain of fTcSC versus TcTM in breast cancer patients scheduled to undergo SLN biopsy. Before initiation, Institutional Review Board approval was obtained and the trial was registered at clinicaltrials.gov (NCT02065232). Female patients, aged $\geq 18$ years with a diagnosis of primary breast cancer or ductal carcinoma in situ (DCIS) with planned SLN biopsy as part of the surgical plan were approached at preoperative clinic visits of the principal investigator (AMW). Pregnant patients, patients undergoing bilateral SLN biopsy, or patients with clinical and/or radiological evidence of metastatic lymph nodes or systemic disease were excluded.

Data on patient age, body mass index (BMI), race, history of diabetes, history of daily narcotic use, cancer treatment involving neoadjuvant chemotherapy or endocrine therapy, use of preoperative needle localization for guided surgical resection, and injecting radiologist were recorded.

\section{Randomization}

Randomization was performed by the Department of Biostatistics at the UCSD Clinical and Translational Research Institute (CTRI). Patients were allocated in 1:1 ratio between fTcSC and TcTM. After a patient consented to the study, the Clinical Trials Office (CTO) assigned a study number to the patient corresponding to either of the two groups. The CTO facilitated ordering of the proper solution for injection with the nuclear technician such that both the radiologist and the operating team were blinded to the pharmaceutical agent injected.

\section{Radiopharmaceutical Preparation}

Both Technetium-99m Sulfur Colloid and TcTM were prepared according to manufacturer package inserts (TcSCPharmulence Inc., Billeria, MA, TcTM-Navidea Pharmaceuticals, Dublin, $\mathrm{OH}$ ) by a centralized radiopharmacy (Cardinal Health, San Diego CA). The TcSC preparation was filtered $(100 \mathrm{~nm})$ by a standard Cardinal Health protocol to produce filtered Technetium-99m Sulfur Colloid (fTcSC). The $\mathrm{pH}$ of the study agents was checked periodically and found to be 6.0 for both fTcSC and TcTM. The study agents were delivered in a 27-gauge insulin syringe with a label that hid the agent identification (the correct drug was verified by the pharmacist, the CTO, and nuclear technician prior to handing the drug to the injecting radiologist with the blinded label).

\section{Injection}

Patients received a $0.1-\mathrm{ml}$ solution $(0.36-0.55 \mathrm{mCi})$ of either fTcSC or TcTM. The patient and radiologist administering the agent were blinded to the injection drug. The injection was performed utilizing a single, intradermal injection overlying the biopsy area or tumor by one of two nuclear medicine radiologists. Immediately before injection, an alcohol swab was used to clean the injection site. The injection time was standardized for $5 \mathrm{~s}$ and intradermal injection was confirmed by presence of a skin wheal.

\section{Background of Pain Questionnaires}

The visual analog scale (VAS) is a measure of pain intensity. ${ }^{8}$ Operationally, the VAS is a horizontal line, $100 \mathrm{~mm}$ in length, anchored by word descriptors at each end. The left and right ends are labeled "no pain" and "worst possible pain," respectively. The patient is instructed to make a vertical mark on the horizontal line that they feel represents their pain intensity. The VAS is scored manually by measuring in millimeters from the left hand end of the line to the point that the patient marks. The short-form McGill Pain Questionnaire (SF-MPQ) is a validated pain survey designed to measure the sensory and affective quality of pain. ${ }^{9}$ The survey consists of 15 descriptors (11 sensory and 4 affective) that may characterize the patient's quality of their pain. Each descriptor is given a rating of $0-3$ corresponding to the patient's response of none, mild, moderate, or severe. The scores for each descriptor are added such that each patient has a sensory score rated from 0 to 33 , an affective score rated 0 to 12 , and a combined score rated 0 to 45 .

\section{Pain Survey Administration}

The VAS and SF-MPQ were administered during the study. The VAS was administered immediately preinjection and then at 1-, 2-, 3-, 4-, and 5-min postinjection time points. The SF-MPQ was administered immediately preinjection and then at $5 \mathrm{~min}$ postinjection. At the 5 -min time point, the patients were instructed to answer the SFMPQ based on the experience during the time encompassing injection and the $5 \mathrm{~min}$ after injection, whereas at preinjection they were instructed to answer based on their current pain. The individual administering the pain surveys was blinded to the study agent. A stopwatch was used to 
keep track of time postinjection and the clock was started when the needle was removed from the patient's skin.

\section{Preoperative Needle Localization Procedure}

For patients scheduled to undergo breast conservation (BCT) surgery, standard practice at our institution is for our breast radiologists to place needles under ultrasound, mammographic, or MRI guidance to aid with surgical resection a few hours prior to planned surgical resection. The procedure is performed in the radiology suite, and when concluded, the patient is immediately brought to the nuclear medicine room for the lymphoscintigraphy procedure.

\section{Endpoints}

The primary endpoint of this study was the assessment of mean changes in VAS pain scores at 1 min postinjection $\left(\mathrm{VAS}_{t=1 \text { min }}-\mathrm{VAS}_{t=0 \mathrm{~min}}\right)$ between fTcSC and TcTM. Secondary endpoints included comparison of mean VAS pain scores in the first 5 min postinjection as well as differences in sensory and affective experiences as captured by the SF-MPQ at $5 \mathrm{~min}$ postinjection between the two groups.

\section{Sample Size Determination and Statistical Analysis}

Calculation of sample size was performed using the assumption that the minimal clinically significant change in VAS score is $13 \mathrm{~mm}$ with an expected standard deviation of $12 .^{10}$ Controlling for probability of a type 1 error, $(\alpha)=0.01$ and power $(1-\beta)=0.9,52$ total patients $(26$ per group) were needed to detect no difference between groups. Trial end was set when a total of 52 patients enrolled and completed the lymphoscintigraphy procedure.

For our primary endpoint, a two-sample $t$ test was used to assess mean differences in pain score changes on the VAS scale at 1 min postinjection between fTcSC and TcTM. The 5-min SF-MPQ scores were analyzed using a two-sample $t$ test to assess mean differences between the groups. Baseline patient characteristics were analyzed with ANOVA for continuous variables or Fisher's exact test/Chi squared tests for categorical variables.

To assess pain differences between the groups over the first 5 min postinjection, we fit a linear mixed-effects model (LMM). The LMM is a random coefficient model that takes both baseline heterogeneity and time-variant effects into consideration by incorporating the subjectspecific intercept and slope. The form of the model is as follows: $Y_{i j}=\beta_{0}+\beta^{\mathrm{T}} X_{i j}+u_{i}^{\mathrm{T}} Z_{i}+e_{i j}$ where $Y_{i j}$ is the change in pain for patient $i$ between minute $j$ and baseline, $\beta_{0}$ is a shared intercept term, $\beta^{\mathrm{T}}$ represents the transpose of the vector whose elements are values of the fixed effects (group, time, their interaction, use of needle localization, and baseline score), $X_{i j}$ is the design matrix for the fixed effects, $u_{i}^{\mathrm{T}}$ represents the transpose of the vector containing the random effects associated with each patient (following a multivariate normal distribution with mean 0 and variance matrix $\Sigma_{u}^{2}$ ), $Z_{i}$ is the design matrix for the random effects, and $e_{i j}$ is a normal distributed error term with mean 0 and variance $\sigma^{2}$. The difference of pain change between two groups for each minute is reported by using contrast tests with approximate normal distribution. The significance level of the contrast tests is adjusted by using the Holm-Bonferroni method to control for false discovery rate for multiple comparisons. ${ }^{11}$

Statistical analysis was performed using R (v3.1.2) software under supervision of the UCSD CTRI. A $p$ value $<0.05$ was considered statistically significant

\section{RESULTS}

Between March 2014 and February 2015, 57 patients were enrolled and provided written consent to participate in the study. A participant flow sheet is seen in Fig. 1. Fiftytwo women underwent successful radiopharmaceutical injection and completed the pain surveys, 27 for fTcSC, and 25 for TcTM. Of the five patients who did not undergo study completion, three patients withdrew prior to the procedure, one patient's treatment plan changed to not include SLN biopsy, and one patient received an incorrect dose of a study agent. There were no statistically significant differences in baseline patient characteristics between the two groups (Table 1).

\section{Pain at 1 Minute PostInjection}

At 1 min postinjection, patients receiving fTcSC experienced a statistically significant higher change in mean pain of $16.8 \mathrm{~mm}$ (standard deviation (SD) 19.5) compared with $0.2 \mathrm{~mm}$ (SD 7.3) in TcTM $(p=0.0002)$. Individual pain scores at 1 min postinjection are depicted in Fig. 2.

\section{SF-MPQ at 5 Min Postinjection}

At 5 min postinjection, there was no statistically significant difference in SF-MPQ pain scores between the groups. The mean total score on the SF-MPQ was 2.8 (SD $3.0)$ in $\mathrm{fTcSC}$ versus 2.1 (SD 2.5) for $\operatorname{TcTM}(p=0.36)$.

\section{Pain During Initial 5 Min Postinjection}

In our final LMM, injection agent $(p<0.001)$, time $(p<0.001)$, and their interaction $(p<0.001)$ were found 
FIG. 1 Patient flow diagram. $S L N$ sentinel lymph node; $f T c S C$ filtered Technetium-99m Sulfur Colloid; TcTM Technetium-99m Tilmanocept

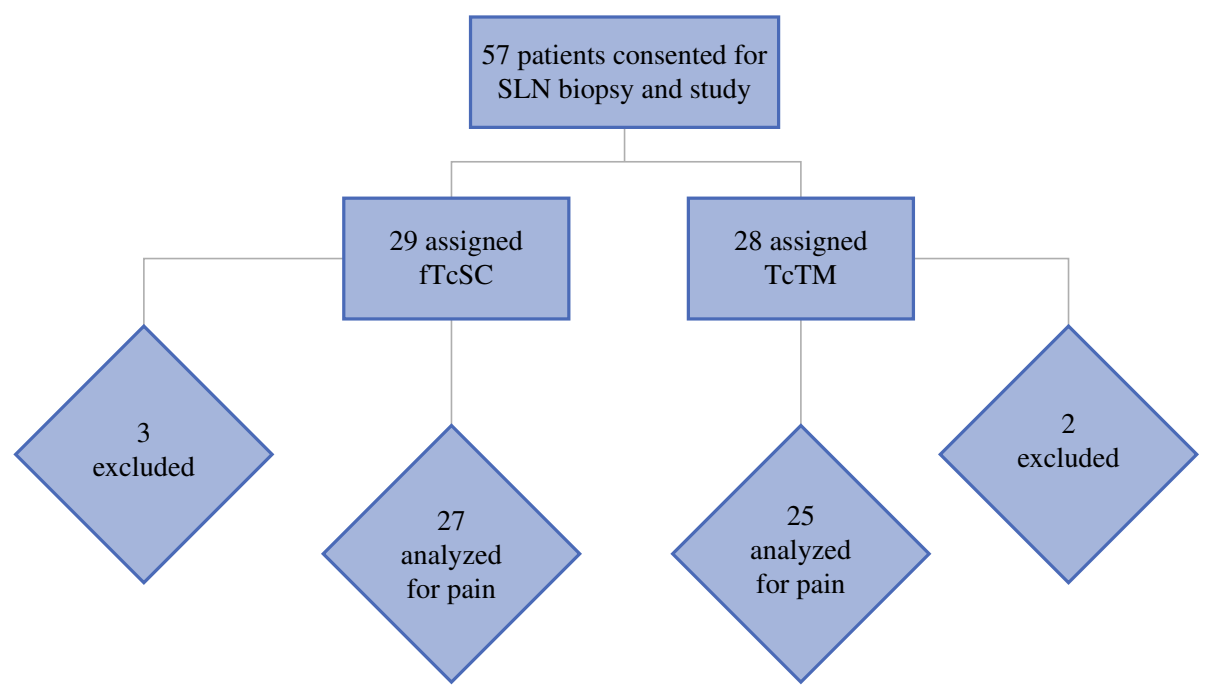

TABLE 1 Baseline patient characteristics

\begin{tabular}{|c|c|c|c|}
\hline & $\begin{array}{l}\mathrm{fTcSC} \\
(n=27)\end{array}$ & $\begin{array}{l}\text { TcTM } \\
(n=25)\end{array}$ & $p$ value* \\
\hline Age (years) & $56.0 \pm 10.4$ & $56.9 \pm 12.7$ & 0.80 \\
\hline BMI $\left(\mathrm{kg} / \mathrm{m}^{2}\right)$ & $26.0 \pm 5.4$ & $26.1 \pm 5.8$ & 0.94 \\
\hline \multicolumn{4}{|l|}{ Race } \\
\hline White & 21 & 23 & 0.23 \\
\hline Asian & 3 & 2 & \\
\hline Hispanic & 3 & 0 & \\
\hline Diabetes & 1 & 0 & 1.00 \\
\hline Daily narcotic use & 1 & 2 & 1.00 \\
\hline \multicolumn{4}{|l|}{ Neoadjuvant treatment } \\
\hline Chemotherapy & 6 & 5 & 0.30 \\
\hline Endocrine & 3 & 0 & \\
\hline $\begin{array}{l}\text { Preoperative needle } \\
\text { localization }\end{array}$ & 18 & 16 & 1.00 \\
\hline \multicolumn{4}{|l|}{ Radiologist } \\
\hline$\# 1$ & 13 & 7 & 0.16 \\
\hline$\# 2$ & 14 & 18 & \\
\hline $\begin{array}{l}\text { Preinjection VAS score } \\
\quad(\mathrm{mm})\end{array}$ & $9.0 \pm 16.0$ & $9.0 \pm 14.1$ & 1.00 \\
\hline SF-MPQ preinjection score & $1.9 \pm 3.4$ & $1.7 \pm 2.9$ & 0.88 \\
\hline
\end{tabular}

Data are presented as mean \pm standard deviation or numbers unless otherwise indicated

fTcSC filtered Technetium-99m Sulfur Colloid; TcTM Technetium99m Tilmanocept, VAS visual analogue scale; $S F-M P Q$ short-form McGill Pain Questionnaire

* ANOVA or $\chi^{2} /$ Fisher's exact test

to be significantly associated with change in pain over the 5 min postinjection period. Preoperative needle localization $(p=0.26)$ and baseline pain scores $(p=0.17)$ were not a significant predictor of changes in pain score. The final model with pain score predictions over the first $5 \mathrm{~min}$ postinjection is depicted in Fig. 3. Furthermore, the model found $\mathrm{fTCSC}$ resulted in significantly more pain of $15.2 \mathrm{~mm}(p<0.001), 11.3 \mathrm{~mm}(p=0.001)$, and $7.5 \mathrm{~mm}$ $(p=0.013)$ at 1,2 , and 3 min postinjection, respectively (Table 2). Differences at 4 and 5 min postinjection were not statistically significant.

\section{DISCUSSION}

Our study is the first to evaluate the difference in pain experienced by patients receiving either an injection of fTcSC versus TcTM. We found that patients receiving fTcSC experience statistically significantly more pain than those receiving TcTM at $1 \mathrm{~min}$ postinjection. Additionally, our LMM incorporating pain scores from the VAS scale demonstrates that patients receiving fTcSC experience significantly more pain during the initial 3 min postinjection compared with TcTM. While the majority of the patients experienced a mild amount of discomfort, a few patients reached the moderate range ( $>40 \mathrm{~mm}$ change) in the $\mathrm{fTcSC}$, whereas none of the subjects in the TcTM group reached the moderate range.

Total pain scores utilizing the SF-MPQ did not differ between groups preinjection or at the 5-min postinjection time points. Although the patients were instructed to answer based on their experience over the previous 5-min postinjection period, at $5 \mathrm{~min}$ the majority of patients had stopped experiencing any pain above baseline in either group and the SF-MPQ may have not adequately reflected their peak pain experience. Additionally, while the SFMPQ has an affective domain that measures distress associated with the injection, with the pain lasting for such 


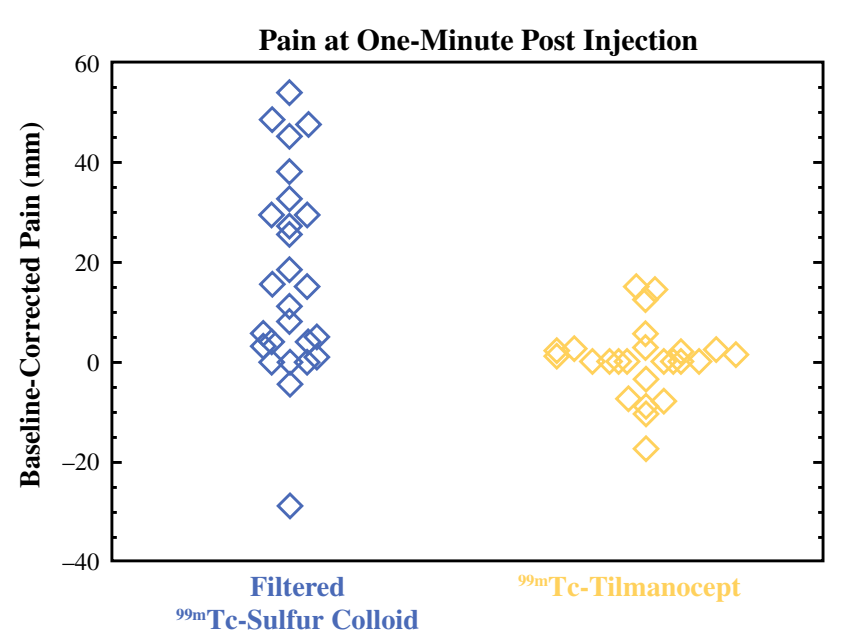

FIG. 2 Baseline-corrected pain 1 min postinjection. This figure represents the pain difference for each individual 1-min after radiopharmaceutical injection

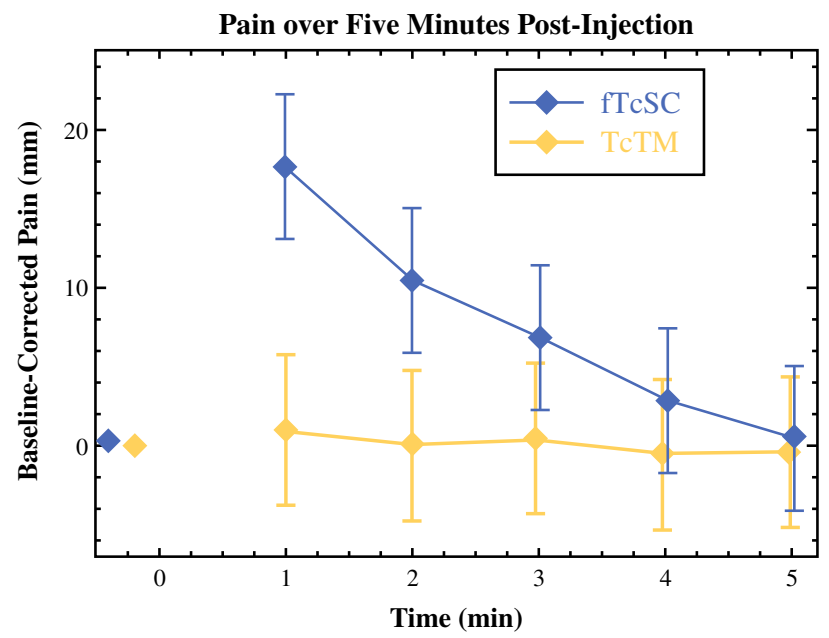

FIG. 3 Linear mixed-effects model. $f T c S C$ filtered Technetium-99m Sulfur Colloid; TcTM Technetium-99m Tilmanocept. This figure represents the final linear mixed-effects model that incorporates injection agent, time, injection $\times$ time interaction, baseline pain score, and needle localization on pain scores baseline-corrected during the first $5 \mathrm{~min}$ postinjection. The diamonds represent mean predicted pain scores for each injection agent with corresponding standard deviation

TABLE 2 Change in VAS pain scores over the 5-min postinjection period

\begin{tabular}{lrrcc}
\hline \multirow{2}{*}{ Time (min) } & \multicolumn{2}{l}{ Change in pain $(\mathrm{mm})^{*}$} & Difference & $p$ value** \\
\cline { 2 - 3 } & \multicolumn{1}{l}{$\mathrm{fTcSC}$} & \multicolumn{1}{c}{ TcTM } & & \\
\hline 1 & $15.7 \pm 5.3$ & $0.5 \pm 5.5$ & 15.2 & $<0.001$ \\
2 & $11.5 \pm 4.3$ & $0.2 \pm 4.4$ & 11.3 & 0.001 \\
3 & $7.3 \pm 3.7$ & $-0.1 \pm 3.8$ & 7.5 & 0.013 \\
4 & $3.1 \pm 3.6$ & $-0.5 \pm 3.7$ & 3.6 & 0.32 \\
5 & $-1.1 \pm 4.1$ & $-0.8 \pm 4.2$ & -0.3 & 0.92 \\
\hline
\end{tabular}

fTcSC filtered Technetium-99m Sulfur Colloid; TcTM Technetium$99 \mathrm{~m}$ Tilmanocept; $V A S$ visual analogue scale

* Mean \pm standard deviation; ** adjusted $p$ value by Holm-Bonferroni method a short duration, we would not expect to see any distress even with pain that reaches moderate levels.

Several studies have verified that injection of TcSC formulations prior to lymphoscintigraphy is painful and have evaluated ways to minimize the pain associated with $\mathrm{TcSC}$ injection. Stojadinovic et al. examined a variety of techniques that involved addition of local anesthetic and sodium bicarbonate to TcSC. They found that adding $1 \%$ lidocaine decreased patient pain, but changing $\mathrm{pH}$ did not alter pain scores. ${ }^{2}$ Hawkins et al. found that a separate injection of lidocaine prior to intradermal injection with TcSC decreased patient pain. ${ }^{12}$ Another study by O'Connor et al. had patients apply topical anesthetic creams prior to TcSC injection. ${ }^{3}$ They did not find benefit with the 
anesthetic creams; however, patients were not asked about their pain until 2-4 days after the procedure. Despite the benefit of either a preinjection lidocaine injection or lidocaine-TcSC solution, neither practice has been universally accepted or adopted likely due to the increased work for the patient or additional preparation for the clinical treatment team.

It is unclear how fTcSC induces significantly more pain than TcTM at the injection site. The $\mathrm{pH}$ of the study agents does not appear to be a factor in the cause of pain as the $\mathrm{pH}$ of both study agents was 6.0. Additionally, the Stojadinovic study found that pain scores did not differ when they altered the $\mathrm{pH}$ of the injected TcSC agent. ${ }^{2}$ In our study, TcSC was filtered with a $100-\mathrm{nm}$ porous filter and the average diameter of injected TcTM was $7 \mathrm{~nm}^{4}$ The dermis receives distal terminations of $A \beta$ and $A \delta$ myelinated fibers. These fibers transfer mechanical stimuli from corpusculated receptors (A $\beta)$ and painful stimuli elicited in free nerve endings (A $\delta) .{ }^{13}$ The larger particle size of the fTcSC may increase the stretch on nociceptive pain receptors in the dermis leading to an intensified pain experience.

Previous literature supports the effectiveness of TcTM as a SLN mapping agent in breast, melanoma, and head and neck cancers. ${ }^{6,7,14-16}$ Our trial demonstrates that patients experience significantly less pain with TcTM compared with fTcSC when standard preparations are injected into the breast for the purpose of SLN mapping. Thus, TcTM may be a more ideal diagnostic radiopharmaceutical, because it minimizes patient discomfort while allowing for effective SLN mapping. Whereas our study only evaluated injection of the agent in the breast, the decreased pain effect may be even more pronounced and desirable when injecting sensitive areas such as the face, mouth, or genitalia as in SLN mapping for other types of cancer. Further study is needed to examine other differentiating factors between the two agents such as cost, availability, and overall efficacy.

There are a few limitations to this trial. First, not all patients received preoperative needle localization. However, in our mixed effects model, neither preoperative baseline pain nor undergoing needle localization significantly affected pain score change from baseline. Additionally, while an intradermal radiopharmaceutical injection was used throughout, the location on the breast was not standardized. Strengths of our study include its randomized, prospective nature, the blinding of the patient and injecting physician to the injected study agent, and use of highly verified pain questionnaires.

\section{CONCLUSIONS}

Injection with $\mathrm{fTcSC}$ causes significantly more pain during the first 3 min after radiopharmaceutical injection compared with TcTM in women undergoing lymphoscintigraphy for breast cancer.

ACKNOWLEDGMENT The authors thank the Clinical Trials Research Institute Biostatistics Department at University of California, San Diego for their assistance with data analysis and interpretation. They also thank Dr. Paul Scheibe for his review and helpful discussion of the manuscript. Navidea Biopharmaceuticals provided funding for administrative assistance in conducting the trial.

DISCLOSURE DRV is the inventor of Tilmanocept and paid consultant of Navidea Biopharmaceuticals.

\section{REFERENCES}

1. Fetzer S, Holmes S. Relieving the pain of sentinel lymph node biopsy tracer injection. Clin J Oncol Nurs. 2008;12:668-70.

2. Stojadinovic A, Peoples GE, Jurgens JS, Howard RS, Schuyler B, Kwon $\mathrm{KH}$, et al. Standard versus $\mathrm{pH}$-adjusted and lidocaine supplemented radiocolloid for patients undergoing sentinellymph-node mapping and biopsy for early breast cancer (PASSION-P trial): a double-blind, randomised controlled trial. Lancet Oncol 2009;10:849-54.

3. O'Connor JM, Helmer SD, Osland JS, Cusick TE, Tenofsky PL. Do topical anesthetics reduce periareolar injectional pain before sentinel lymph node biopsy? Am J Surg 2011;202:707-11; discussion 711-2.

4. Wallace AM, Hoh CK, Vera DR, Darrah DD, Schulteis G. Lymphoseek: a molecular radiopharmaceutical for sentinel node detection. Ann Surg Oncol. 2003;10:531-8.

5. Vera DR, Wallace AM, Hoh CK, Mattrey RF. A synthetic macromolecule for sentinel node detection: ${ }^{99 \mathrm{~m}} \mathrm{Tc}-\mathrm{DTPA}-\mathrm{man}-$ nosyl-dextran. J Nucl Med. 2001;42:951-9.

6. Wallace AM, Han LK, Povoski SP, Deck K, Schneebaum S, Hall $\mathrm{NC}$, et al. Comparative evaluation of $\left[{ }^{99 \mathrm{~m}} \mathrm{Tc}\right]$ tilmanocept for sentinel lymph node mapping in breast cancer patients: results of two phase 3 trials. Ann Surg Oncol. 2013;20:2590-9.

7. Sondak VK, King DW, Zager JS, Schneebaum S, Kim J, Leong SP, et al. Combined analysis of phase III trials evaluating $\left[{ }^{99 \mathrm{~m}} \mathrm{Tc}\right]$ tilmanocept and vital blue dye for identification of sentinel lymph nodes in clinically node-negative cutaneous melanoma. Ann Surg Oncol. 2013;20:680-8.

8. Bijur PE, Silver W, Gallagher EJ. Reliability of the visual analog scale for measurement of acute pain. Acad Emerg Med. 2001;8:1153-7.

9. Melzack R. The McGill Pain Questionnaire: major properties and scoring methods. Pain. 1975;1:277-99.

10. Todd KH, Funk KG, Funk JP, Bonacci R. Clinical significance of reported changes in pain severity. Ann Emerg Med. 1996;27:485-9.

11. Holm S. A simple sequentially rejective multiple test procedure. Scand J Statist. 1979;6:65-70.

12. Hawkins AS, Yoo DC, Movson JS, Noto RB, Powers K, Baird G, et al. Administration of subcutaneous buffered lidocaine prior to breast lymphoscintigraphy reduces pain without decreasing lymph node visualization. J Nucl Med Technol. 2014;42:260-4.

13. Provitera V, Nolano M, Pagano A, Caporaso G, Stancanelli A, Santoro L. Myelinated nerve endings in human skin. Muscle Nerve. 2007;35:767-75.

14. Agrawal A, Civantos FJ, Brumund KT, Chepeha DB, Hall NC, Carroll WR, et al. [ $\left.{ }^{99 \mathrm{~m}} \mathrm{Tc}\right]$ Tilmanocept accurately detects sentinel lymph nodes and predicts node pathology status in patients with oral squamous cell carcinoma of the head and neck: results of a 
phase III multi-institutional trial. Ann Surg Oncol. 2015. doi:10. 1245/s10434-015-4382-x.

15. Baker JL, Pu M, Tokin CA, Hoh CK, Vera DR, Messer K, et al. Comparison of $\left[{ }^{99 \mathrm{~m}} \mathrm{Tc}\right]$ tilmanocept and filtered $\left[{ }^{99 \mathrm{~m}} \mathrm{Tc}\right]$ sulfur colloid for identification of SLNs in breast cancer patients. Ann Surg Oncol. 2015;22:40-5.

16. Tokin CA, Cope FO, Metz WL, Blue MS, Potter BM, Abbruzzese $\mathrm{BC}$, et al. The efficacy of Tilmanocept in sentinel lymph mode mapping and identification in breast cancer patients: a comparative review and meta-analysis of the ${ }^{99 \mathrm{~m}} \mathrm{Tc}$-labeled nanocolloid human serum albumin standard of care. Clin Exp Metastasis. 2012;29:681-6. 